\title{
What's Eating You? Human Flea (Pulex irritans)
}

\author{
Megan O’Donnell, BS; Dirk M. Elston, MD
}

\section{PRACTICE POINTS}

- The human flea, Pulex irritans, is a vector for various human diseases including the bubonic plague, bartonellosis, and rickettsioses.

- Presenting symptoms of flea bites include intensely pruritic, urticarial to vesicular papules on exposed areas of skin.

- The primary method of flea control includes a combination of insecticidal products and insect growth regulators.
The human flea, Pulex irritans, is an important vector in the transmission of human diseases. This flea has been identified as a vector for the bubonic plague as far back as the 14th century, causing millions of deaths. Pulex irritans also has been identified as a vector for Bartonella bacteria species, which can cause trench fever. Rickettsia species, which can cause Rocky Mountain spotted fever and Mediterranean spotted fever, also have been identified in $P$ irritans. Flea bites present as intensely pruritic papules and can be treated with topical antipruritics and corticosteroids. The most effective form of flea control and prevention includes a combination of insecticidal products and insect growth regulators.

Cutis. 2020;106:233-235.

\section{Characteristics}

The ubiquitous human flea, Pulex irritans, is a hematophagous wingless ectoparasite in the order Siphonaptera (wingless siphon) that survives by consuming the blood of its mammalian and avian hosts. Due to diseases such as the bubonic plague, fleas have claimed more victims than all the wars ever fought; in the 14th century, the Black Death caused more than 200 million deaths. Fleas fossilized in amber have been found to be 200 million years old and closely resemble the modern human flea, demonstrating the resilience of the species.

The adult human flea is a small, reddish brown, laterally compressed, wingless insect that is approximately 2- to 3.5-mm long (females, 2.5-3.5 mm; males, 2-2.5 mm) and enclosed by a tough cuticle. Compared to the dog flea (Ctenocephalides canis) and cat flea (Ctenocephalides felis), P irritans has no combs or ctenidia (Figure 1). Fleas have large powerful hind legs enabling them to jump horizontally or vertically 200 times their body length (equivalent to a 6-foot human jumping 1200 feet) using

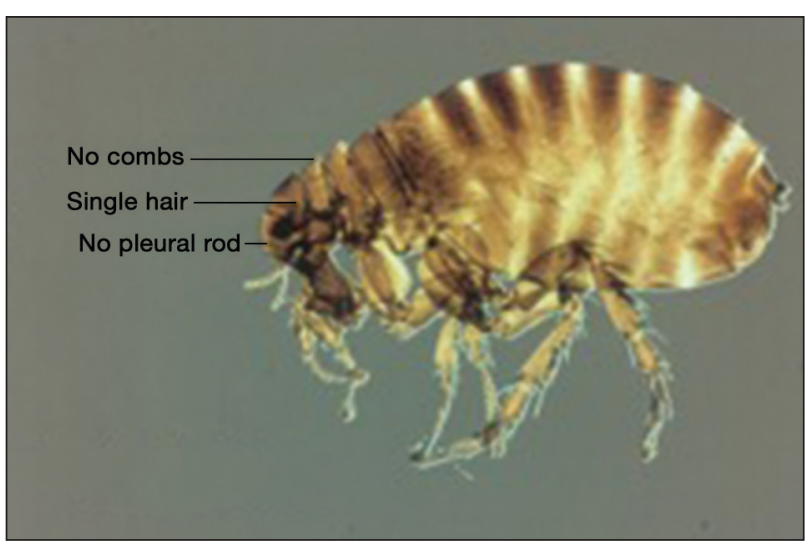

FIGURE 1. Pulex irritans anatomy. A reddish brown flea lacking characteristic features from most other flea species including a comb and pleural rod.

Ms. O'Donnell is from Sidney Kimmel Medical College, Thomas Jefferson University, Philadelphia, Pennsylvania. Dr. Elston is from the Department of Dermatology and Dermatologic Surgery, Medical University of South Carolina, Charleston.

The authors report no conflict of interest.

The images are in the public domain.

Correspondence: Megan O’Donnell, BS, 1025 Walnut St \#100, Philadelphia, PA 19107 (mco003@jefferson.edu).

doi:10.12788/cutis.0107 
stored muscle energy in a pad on the hind legs composed of the elastic protein resilin. ${ }^{1}$ They feed off a wide variety of hosts, including humans, pigs, cats, dogs, goats, sheep, cattle, chickens, owls, foxes, rabbits, mice, and feral cats. The flea's mouthparts are highly specialized for piercing the skin and sucking its blood meal via direct capillary cannulation.

\section{Life Cycle}

There are 4 stages of the flea life cycle: egg, larva, pupa, and adult. Most adult flea species mate on the host; the female will lay an average of 4 to 8 small white eggs on the host after each blood meal, laying more than 400 eggs during her lifetime. The eggs then drop from the host and hatch in approximately 4 to 6 days to become larvae. The active larvae feed on available organic matter in their environment, such as their parents' feces and detritus, while undergoing 3 molts within 1 week to several months. ${ }^{2}$ The larva then spins a silken cocoon from modified salivary glands to form the pupa. In favorable conditions, the pupa lasts only a few weeks; however, it can last for a year or more in unfavorable conditions. Triggers for emergence of the adult flea from the pupa include high humidity, warm temperatures, increased levels of carbon dioxide, and vibrations including sound. An adult $P$ irritans flea can live for a few weeks to more than 1.5 years in favorable conditions of lower air temperature, high relative humidity, and access to a host. ${ }^{3}$

\section{Related Diseases}

Pulex irritans can be a vector for several human diseases. Yersinia pestis is a gram-negative bacteria that causes plague, a highly virulent disease that killed millions of people during its 3 largest human pandemics. The black rat (Rattus rattus) and the oriental rat flea (Xenopsylla cheopis) have been implicated as initial vectors; however, transmission may be human-to-human with pneumonic plague, and septicemic plague may be spread via Pulex fleas or body lice. ${ }^{4,5}$ In 1971, Y pestis was isolated from $P$ irritans on a $\operatorname{dog}$ in the home of a plague patient in Kayenta, Arizona. ${ }^{6}$ Yersinia pestis bacterial DNA also was extracted from $P$ irritans during a plague outbreak in Madagascar in $2014^{7}$ and was implicated in epidemiologic studies of plague in Tanzania from 1986 to 2004, suggesting it also plays a role in endemic disease. ${ }^{8}$

Bartonellosis is an emerging disease caused by different species of the gram-negative intracellular bacteria of the genus Bartonella transmitted by lice, ticks, and fleas. Bartonella quintana causes trench fever primarily transmitted by the human body louse, Pediculus humanus corporis, and resulted in more than 1 million cases during World War I. Trench fever is characterized by headache, fever, dizziness, and shin pain that lasts 1 to 3 days and recurs in cycles every 4 to 6 days. Other clinical manifestations of $B$ quintana include chronic bacteremia, endocarditis, lymphadenopathy, and bacillary angiomatosis. ${ }^{9}$ Bartonella henselae causes cat scratch fever, characterized by lymphadenopathy, fever, headache, joint pain, and lethargy from infected cat scratches or the bite of an infected flea. Bartonella rochalimae also has been found to cause a trench fever-like bacteremia. ${ }^{10}$ Bartonella species have been found in P irritans, and the flea is implicated as a vector of bartonellosis in humans. ${ }^{11-15}$

Rickettsioses are worldwide diseases caused by the gram-negative intracellular bacteria of the genus Rickettsia transmitted to humans via hematophagous arthropods. The rickettsiae traditionally have been classified into the spotted fever or typhus groups. The spotted fever group (ie, Rocky Mountain spotted fever, Mediterranean spotted fever) is transmitted via ticks. The typhus group is transmitted via lice (epidemic typhus) and fleas (endemic or murine typhus). Murine typhus can be caused by Rickettsia typhi in warm coastal areas around the world where the main mammal reservoir is the rat and the rat flea vector $X$ cheopis. Clinical signs of infection are abrupt onset of fever, headaches, myalgia, malaise, and chills, with a truncal maculopapular rash progressing peripherally several days after the initial clinical signs. Rash is present in up to $50 \%$ of cases. ${ }^{16}$ Rickettsia felis is an emerging flea-borne pathogen causing an acute febrile illness usually transmitted via the cat flea $C$ felis. ${ }^{17}$ Rickettsia species DNA have been found to be present in $P$ irritans from dogs ${ }^{18}$ and livestock ${ }^{19}$ and pose a risk for causing rickettsioses in humans.

\section{Environmental Treatment and Prevention}

Flea bites present as intense, pruritic, urticarial to vesicular papules that usually are located on the lower extremities but also can be present on exposed areas of the upper extremities and hands (Figure 2). Human fleas infest clothing, and bites can be widespread. Topical antipruritics and corticosteroids can be used for controlling itch and the intense cutaneous inflammatory response. The flea host should be identified in areas of the home, school, farm, work, or local environment. House pets should be examined and treated by a veterinarian. The pet's

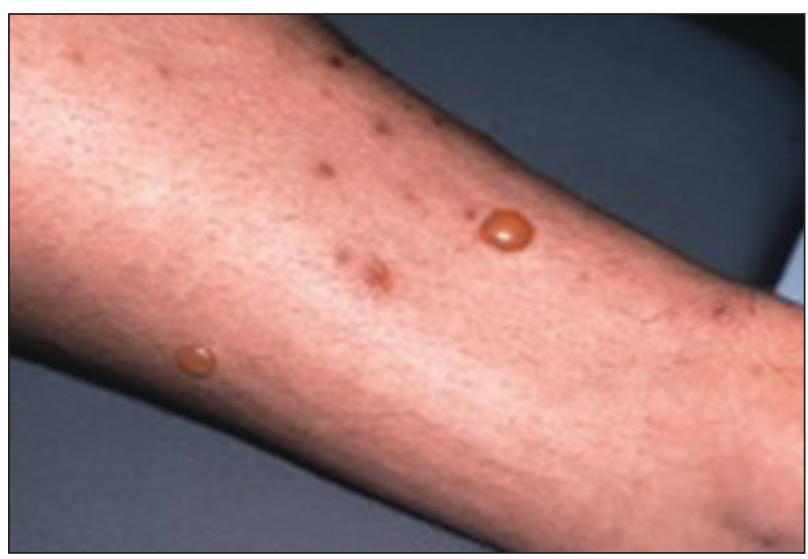

FIGURE 2. Vesicular papules on an exposed area of the arm from flea bites (Pulex irritans). 
bedding should be washed and dried at high temperatures, and carpets and floors should be routinely vacuumed or cleaned to remove eggs, larvae, flea feces, and/or pupae. The killing of adult fleas with insecticidal products (eg, imidacloprid, fipronil, spinosad, selamectin, lufenuron, ivermectin) is the primary method of flea control. Use of insect growth regulators such as pyriproxyfen inhibits adult reproduction and blocks the organogenesis of immature larval stages via hormonal or enzymatic actions. ${ }^{20}$ The combination of an insecticide and an insect growth regulator appears to be most effective in their synergistic actions against adult fleas and larvae. There have been reports of insecticidal resistance in the flea population, especially with pyrethroids. ${ }^{21,22}$ A professional exterminator and veterinarian should be consulted. In recalcitrant cases, evaluation for other wild mammals or birds should be performed in unoccupied areas of the home such as the attic, crawl spaces, and basements, as well as inside walls.

\section{Conclusion}

The human flea, $P$ irritans, is an important vector in the transmission of human diseases such as the bubonic plague, bartonellosis, and rickettsioses. Flea bites present as intensely pruritic, urticarial to vesicular papules that most commonly present on the lower extremities. Flea bites can be treated with topical steroids, and fleas can be controlled by a combination of insecticidal products and insect growth regulators.

\section{REFERENCES}

1. Burrow M. How fleas jump. J Exp Biol. 2009;18:2881-2883.

2. Buckland PC, Sandler JP. A biogeography of the human flea, Pulex irritans L (Siphonaptera: Pulicidae). J Biogeogr. 1989;16:115-120.

3. Krasnov BR. Life cycles. In: Krasnov BR, ed. Functional and Evolutional Ecology of Fleas. Cambridge, MA: Cambridge Univ Press; 2008:45-67.

4. Dean KR, Krauer F, Walloe L, et al. Human ectoparasites and the spread of plague in Europe during the second pandemic. Proc Natl Acad Sci U S A. 2018;115:1304-1309.
5. Hufthammer AK, Walloe L. Rats cannot have been intermediate hosts for Yersinia pestis during medieval plague epidemics in Northern Europe. J Archeol Sci. 2013;40:1752-1759.

6. Archibald WS, Kunitz SJ. Detection of plague by testing serums of dogs on the Navajo Reservation. HSMHA Health Rep. 1971;86:377-380.

7. Ratovonjato J, Rajerison M, Rahelinirina S, et al. Yersinia pestis in Pulex irritans fleas during plague outbreak, Madagascar. Emerg Infect Dis. 2014;20:1414-1415

8. Laudisoit A, Leirs H, Makundi RH, et al. Plague and the human flea, Tanzania. Emerg Infect Dis. 2007;13:687-693.

9. Foucault C, Brouqui P, Raoult D. Bartonella quintana characteristics and clinical management. Emerg Infect Dis. 2006;12:217-223.

10. Eremeeva ME, Gerns HL, Lydy SL, et al. Bacteremia, fever, and splenomegaly caused by a newly recognized bartonella species. $N$ Engl J Med. 2007; 356:2381-2387.

11. Marquez FJ, Millan J, Rodriguez-Liebana JJ, et al. Detection and identification of Bartonella sp. in fleas from carnivorous mammals in Andalusia, Spain. Med Vet Entomol. 2009;23:393-398.

12. Perez-Martinez L, Venzal JM, Portillo A, et al. Bartonella rochalimae and other Bartonella spp. in fleas, Chile. Emerg Infect Dis. 2009;15:1150-1152.

13. Sofer S, Gutierrez DM, Mumcuoglu KY, et al. Molecular detection of zoonotic bartonellae (B. henselae, B. elizabethae and B. rochalimae) in fleas collected from dogs in Israel. Med Vet Entomol. 2015;29:344-348.

14. Zouari S, Khrouf F, M'ghirbiY, et al. First molecular detection and characterization of zoonotic Bartonella species in fleas infesting domestic animals in Tunisia. Parasit Vectors. 2017;10:436.

15. Rolain JM, Bourry, O, Davoust B, et al. Bartonella quintana and Rickettsia felis in Gabon. Emerg Infect Dis. 2005;11:1742-1744.

16. Tsioutis C, Zafeiri M, Avramopoulos A, et al. Clinical and laboratory characteristics, epidemiology, and outcomes of murine typhus: a systematic review. Acta Trop. 2017;166:16-24.

17. Brown L, Macaluso KR. Rickettsia felis, an emerging flea-borne rickettsiosis. Curr Trop Med Rep. 2016;3:27-39.

18. Oteo JA, Portillo A, Potero F, et al. 'Candidatus Rickettsia asemboensis' and Wolbachia spp. in Ctenocephalides felis and Pulex irritans fleas removed from dogs in Ecuador. Parasit Vectors. 2014;7:455.

19. Ghavami MB, Mirzadeh H, Mohammadi J, et al. Molecular survey of ITS spacer and Rickettsia infection in human flea, Pulex irritans. Parasitol Res. 2018;117:1433-1442.

20. Traversa D. Fleas infesting pets in the era of emerging extra-intestinal nematodes. Parasit Vectors. 2013;6:59.

21. Rust MK. Insecticide resistance in fleas. Insects. 2016;7:10.

22. Ghavami MB, Haghi FP, Alibabaei Z, et al. First report of target site insensitivity to pyrethroids in human flea, Pulex irritans (Siphonaptera: Pulicidae). Pest Biochem Physiol. 2018;146:97-105. 